\title{
Can macular xanthophylls replace cholesterol in formation of the liquid-ordered phase in lipid-bilayer membranes?*
}

\author{
Witold K. Subczynski ${ }^{1}$, Anna Wisniewska-Becker ${ }^{2}$ and Justyna Widomska ${ }^{3 凶}$ \\ 'Department of Biophysics, Medical College of Wisconsin, Milwaukee, WI, USA; 2Department of Biophysics, Faculty of Biochemistry, Biophysics, \\ and Biotechnology, Jagiellonian University, Krakow, Poland; ${ }^{3}$ Department of Biophysics, Medical University of Lublin, Lublin, Poland
}

\begin{abstract}
Lateral organization of membranes made from binary mixtures of dimyristoylphosphatidylcholine (DMPC) or dipalmitoylphosphatidylcholine (DPPC) and macular xanthophylls (lutein or zeaxanthin) was investigated using the saturation-recovery (SR) EPR spin-labeling discrimination by oxygen transport (DOT) method in which the bimolecular collision rate of molecular oxygen with the nitroxide spin label is measured. This work was undertaken to examine whether or not lutein and zeaxanthin, macular xanthophylls that parallel cholesterol in its function as a regulator of both membrane fluidity and hydrophobicity, can parallel other structural functions of cholesterol, including formation of the liquid-ordered phase in membranes. The DOT method permits discrimination of different membrane phases when the collision rates (oxygen transport parameter) differ in these phases. Additionally, membrane phases can be characterized by the oxygen transport parameter in situ without the need for separation, which provides information about the dynamics of each phase. In gel-phase membranes, two coexisting phases were discriminated in the presence of macular xanthophylls - namely, the liquid-ordered-like and solid-ordered-like phases. However, in fluid-phase membranes, xanthophylls only induce the solitary liquidordered-like phase, while at similar concentrations, cholesterol induces coexisting liquid-ordered and liquid-disordered phases. No significant differences between the effects of lutein and zeaxanthin were found.
\end{abstract}

Key words: lutein, zeaxanthin, liquid-ordered phase, spin-labeling, EPR

Received: 19 October, 2011; accepted: 01 March, 2012; available on-line: 17 March, 2012

\section{INTRODUCTION}

Rohmer et al. (1979) proposed that in prokaryotes dipolar carotenoids act to reinforce and regulate membrane fluidity similarly to cholesterol in eukaryotes. We were intrigued by this hypothesis, and in our earlier papers, we investigated the effects of dipolar carotenoids on the physical properties of lipid-bilayer membranes. We demonstrated that the effects of dipolar carotenoids (including lutein and zeaxanthin) on membrane structure and dynamics are in many ways similar to the effects of cholesterol. Both membrane modifiers increase order, decrease alkyl-chain motion in fluid-phase membranes, and disorder lipids in gel-phase membranes (Subczynski et al., 1992; Subczynski et al., 1993; Kusumi et al., 1986; Subczynski \& Kusumi, 1986; Pasenkiewicz-Gierula et al., 1990). Both are known to broaden the gel-to-fluid phase transition and increase the mobility of polar head groups
(Subczynski et al., 1989; Wisniewska et al. 2006c). As a rule, the presence of unsaturated alkyl chains moderates the effects of dipolar carotenoids and cholesterol (Subczynski et al., 1989; Kusumi et al., 1986). In saturated membranes, $10 \mathrm{~mol} \%$ of dipolar carotenoids exerts an effect similar to that of $15-20 \mathrm{~mol} \%$ of cholesterol (Subczynski et al. 1992; Subczynski et al. 1993; Gruszecki \& Strzalka, 2005).

There are major differences between the effects of dipolar carotenoids and cholesterol on membrane organization and dynamics. The ordering effect of cholesterol does not depend on bilayer thickness (Kusumi et al., 1986), whereas the relation between the length of the carotenoid molecule and the thickness of the membrane is a significant factor in determining the effect of dipolar carotenoids on membrane properties (Subczynski et al., 1993; Wisniewska et al., 2006c). Intercalation of cholesterol in lipid-bilayer membranes decreases the frequency of vertical fluctuation of the ends of alkyl chains toward the polar head-group region, but increases the frequency of chain-bending in the membrane center (Yin \& Subczynski, 1996; Mainali et al., 2011). Intercalation of dipolar carotenoids in membranes decreases the frequency of alkyl-chain bending at all depths in the lipid bilayer (Yin \& Subczynski, 1996).

Numerous investigators have shown that there is a direct correlation between cellular carotenoid content and stabilization of the bacterial membrane (Chamberlain et al., 1991; Rottem \& Markowitz, 1979). Moreover, bacteria may also modulate membrane fluidity by changing carotenoid synthesis from dipolar to nonpolar and vice versa (Jagannadham et al., 2000; Chattopadhyay et al., 1997). Bacteria, especially when it lives in extreme conditions, should possess stable membranes that provide a high barrier to nonspecific permeation of small molecules (Jagannadham et al., 2000; Britton, 1995) and that protect the cell against environmental physical stress, such as solar radiation, high and low temperatures, and salt. Incorporation of dipolar carotenoids or glycosylated dipolar carotenoids into the membrane serves this purpose well. Dipolar carotenoids stabilize both halves of the lipid bilayer like transmembrane "rivets," increasing membrane rigidity by ordering lipid alkyl chains (Subczynski et al., 1992; Subczynski et al., 1993). We would also like to emphasize that polar carotenoids increase the membrane's hydrophobic barrier to polar molecules and ions (Wisniewska \& Subczynski, 1998), which is one of the most fundamental functions

e-mail: jwidomska@gmail.com

*Presented at the 16th International Symposium on Carotenoids, 17-22 July, 2011, Kraków, Poland

Abbreviations: DMPC, dimyristoylphosphatidylcholine; DPPC, dipalmitoylphosphatidylcholine; DOT, discrimination by oxygen transport; 5-PC, 1-palmitoyl-2-(5-doxylstearoyl)phosphatidylcholine. 
of biological membranes. Detailed hydrophobicity profiles may provide the basis to understand membrane permeability for both polar and non-polar molecules (Wisniewska \& Subczynski, 1998; Subczynski \& Widomska 2009). It should also be noted that monopolar and nonpolar carotenoids (like $\beta$-cryptoxanthin and $\beta$-carotene) affect membrane properties much less than dipolar carotenoids (Wisniewska et al., 2006c).

In spite of the substantial literature on the subject, many questions about carotenoid-membrane interactions have not been clearly answered or even addressed. These include questions concerning the distribution of carotenoids between membrane domains and the lateral organization of carotenoid-containing membranes. Krinsky and coauthors (Krinsky, 2002; Krinsky et al., 2003) observed that the need exists for extensive studies of carotenoid-membrane interactions in model systems to clarify ambiguous results in the literature. We were responsive to these articles: a novel method developed by us, the discrimination by oxygen transport (DOT) method (Subczynski et al., 2007a), was applied to membranes containing carotenoids. We showed that in membranes containing raft domains, the macular xanthophylls, lutein and zeaxanthin, are not distributed uniformly but excluded from saturated raft domains and concentrated in unsaturated bulk lipids (Wisniewska \& Subczynski 2006a, Wisniewska \& Subczynski 2006b). We believe these results are very significant to understand the role of macular xanthophylls in protecting against lipid peroxidation (Wrona et al., 2004; Pintea et al., 2011) in membranes of the eye retina and in preventing age-related macular degeneration (Bone et al., 2001; Landrum et al., 1997).

In this work, we carried out DOT studies on the lateral organization of xanthophyll-containing membranes to examine whether or not lutein and zeaxanthin can parallel other structural functions of cholesterol, including formation of the liquid-ordered $\left(l_{\mathrm{o}}\right)$ phase. Depending on temperature and cholesterol concentration in phospholipidcholesterol mixtures, the $l_{\mathrm{o}}$ phase can coexist with other phases-namely, solid-ordered $\left(s_{0}\right)$ and liquid-disordered $\left(l_{\mathrm{d}}\right)$ - or can form a single $l_{\mathrm{o}}$ phase within the entire membrane (Vist \& Davis, 1990). One of the simplest model membranes that contains the $l_{0}$ phase is a binary mixture of dimyristoylphosphatidylcholine (DMPC) and cholesterol, for which the phase diagram and lipid motion have been well-characterized (Kusumi et al., 1986; Almeida et al, 1992; Almeida et al, 2005; Recktenwald \& McConnell, 1981; Shimshick \& McConnell, 1973). In this work, we simply replaced cholesterol with either lutein or zeaxanthin and carried out DOT measurements for DMPC-xanthophyll and dipalmitoylphosphatidylcholine (DPPC)-xanthophyll membranes below and above the phase-transition temperature. Using the DOT method, we were able to recognize membrane phases induced by the presence of carotenoids. This approach allowed us to study physical characteristics and lipid organization at different depths in the membrane phases as well as measure lipid dynamics. This information was obtained simultaneously for coexisting phases without their separation. In these investigations, we also paid special attention to differences between lutein and zeaxanthin.

\section{MATERIALS AND METHODS}

Materials. Xanthophylls, lutein and zeaxanthin, were purchased from CaroteNature (Lupsingen, Switzerland). DMPC, DPPC, cholesterol, and the spin label 1-palmitoyl-2-(5-doxylstearoyl)phosphatidylcholine (5-PC) (see
Fig. 1 for their structure) were obtained from Avanti Polar Lipids, Inc. (Alabaster, AL USA). Other chemicals, of at least reagent grade, were purchased from SigmaAldrich (Milwaukee, WI USA).

Preparation of phosphatidylcholine-xanthophyll membranes. The membranes used in this work were multilamellar dispersions of investigated phosphatidylcholine (PC) containing $1 \mathrm{~mol} \%$ of 5-PC spin label and various amounts of xanthophylls in the range of their miscibility from 0 to $20 \mathrm{~mol} \%$. Briefly, these membranes were prepared using the film deposition method (Wisniewska \& Subczynski, 1998). Chloroform solutions of lipids, xanthophylls, and spin labels (containing $5 \mu \mathrm{mol}$ of the total lipid) were combined to obtain a desired compound concentration. Chloroform was evaporated with a stream of nitrogen and with the test tube in constant rotation in order to deposit a uniform film of lipid over the bottom of the tube. The lipid film was thoroughly dried under reduced pressure (about $0.1 \mathrm{mmHg}$ ) for $12 \mathrm{~h}$. A buffer solution (10 mM PIPES and $150 \mathrm{mM}$ $\mathrm{NaCl}, \mathrm{pH}$ 7.0) was added to the dried film at a temperature above the phase-transition temperature of the PC membrane and vortexed vigorously. All preparations and measurements with xanthophylls were performed in darkness or dim light and, if possible, under nitrogen.

EPR measurements. Membranes were centrifuged briefly $\left(16000 \times g\right.$ for $15 \mathrm{~min}$ at $\left.4^{\circ} \mathrm{C}\right)$, and the loose pellet was used for EPR measurements. The sample was placed in a capillary $(0.6 \mathrm{~mm}$ i.d.) made of the gaspermeable polymer TPX (Subczynski et al., 2005; Hyde \& Subczynski, 1989). For measurements of the oxygen transport parameter, the concentration of oxygen in the sample was controlled by equilibration with the same gas that was used for the temperature control (i.e., a controlled mixture of nitrogen and dry air adjusted with flowmeters (Matheson Gas Products, model 7631H-604)) (Subczynski et al., 2005; Hyde \& Subczynski, 1989; Kusumi et al., 1982).

The $T_{1} \mathrm{~s}$ (spin-lattice relaxation times) of the spin label were determined by analyzing the saturation recovery (SR) signal of the central line obtained by shortpulse SR EPR at X-band with the use of a loop-gap resonator (Yin et al., 1987; Yin et al., 1990; Subczynski et al., 2005; Yin \& Subczynski, 1996). Typically, $10^{5}$ to $10^{6}$ decays were acquired with 2048 data points on each decay. Sampling intervals were 2, 4, 8, 16, or 32 ns depending on the sample, temperature, and oxygen tension. The total accumulation time was typically 2 to $5 \mathrm{~min}$. SR signals were fitted by single- or doubleexponential functions. When a single-exponential fit was satisfactory, the decay time constant was evaluated with a standard deviation smaller than $\pm 3 \%$ from the mean value for independent experiments (for samples prepared independently). When a double-exponential fit was necessary, and satisfactory, the decay times were usually evaluated with standard deviations less than $\pm 5 \%$ and $\pm 10 \%$ for longer and shorter recovery time constants, respectively.

Outline of theory for the DOT method. In dualprobe SR EPR experiments, molecular oxygen is introduced in the membrane suspension as a relaxation agent. This relaxation agent induces spin exchange, which leads to faster spin-lattice relaxation of the nitroxide. With the DOT method, the observable parameter is the spinlattice relaxation time, $T_{1}$, of lipid spin labels, and the measured value is the bimolecular collision rate between molecular oxygen and the nitroxide moiety of spin labels (Ashikawa et al., 1994, Kawasaki et al., 2001). When located in two different membrane environments, the 


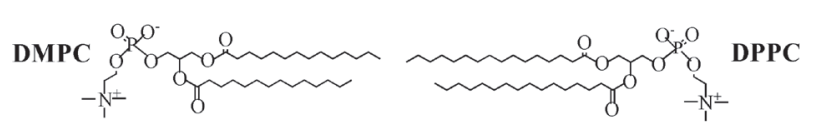

CHOL

$$
\text { HOR }
$$

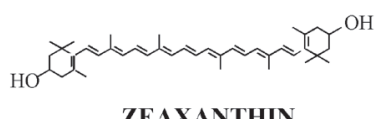

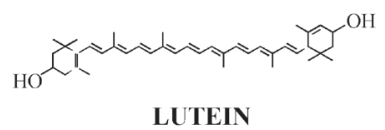

Figure 1. Chemical structures of lutein and zeaxanthin together with the structure of DMPC, DPPC, cholesterol, and 5-PC spin label.

spin label alone most often cannot differentiate between domains, giving very similar (indistinguishable) conventional EPR spectra and similar $T_{1}$ values. However, even small differences in lipid-packing will affect oxygen partitioning and oxygen diffusion, which can be easily detected by observing the different $T_{1}$ s from spin labels in two locations in the presence of molecular oxygen. SR curves from 5-PC located in membranes made from the PCxanthophyll mixture were recorded for samples equilibrated with nitrogen and with a specific partial pressure of oxygen (a mixture of air and nitrogen). The doubleexponential SR signal indicated the presence of two membrane environments. The results were expressed in terms of the oxygen transport parameter, $W$, which is proportional to the local oxygen diffusion-concentration product in the specific membrane domain (Kusumi et al., 1982):

$W=T_{1}^{-1}$ (Air) $-T_{1}^{-1}\left(\mathrm{~N}_{2}\right), W \sim C\left(\mathrm{O}_{2}\right) D\left(\mathrm{O}_{2}\right)$

Here, the $T_{1} \mathrm{~s}$ are the spin-lattice relaxation times of the nitroxides in samples equilibrated with atmospheric air and nitrogen, respectively. $C\left(\mathrm{O}_{2}\right)$ and $D\left(\mathrm{O}_{2}\right)$ are the local oxygen concentration and the local oxygen diffusion coefficient (see Subczynski et al., 2007a for further explanation).

\section{SR measurements of the oxygen transport parameter}

SR measurements for 5-PC in PC-xanthophyll membranes were carried out systematically as a function of xanthophyll concentration, temperature, and the partial pressure of oxygen in the equilibrating gas mixture. All $T_{1}$ values were determined by fitting the SR signals for 5-PC to single and double exponentials. In deoxygenated samples, all SR signals were fitted successfully to single-exponential functions, indicating that the spin label alone cannot discriminate purported phases (data not shown). All SR signals for membranes equilibrated with air, but not containing xanthophylls, were also fitted successfully to single exponentials, indicating that the DOT method does not discriminate coexisting phases (data not shown). Representative SR signals from samples equilibrated with $40 \%$ air for DMPC membranes with $10 \mathrm{~mol} \%$ lutein are shown in Fig. 2. It is clear that below the phase-transition temperature, at $15^{\circ} \mathrm{C}$, the SR signal was fitted successfully to the double-exponential function, indicating the presence of two phases (compare the residual for single- (Fig. 2A) and double-exponential (Fig. 2B) fits). However, above the phase-transition temperature, at $35^{\circ} \mathrm{C}$, successful fits were single exponentials (Fig. 2C). Double-exponential fits were satisfactory only for measurements below the phase-transition temperature of DMPC membrane $\left(23.6^{\circ} \mathrm{C}\right)$ and DPPC membrane $\left(41.2^{\circ} \mathrm{C}\right)$ and for xanthophyll concentrations of 5 and $10 \mathrm{~mol} \%$. All SR signals measured above the phase transition and for xanthophyll concentrations greater than $10 \mathrm{~mol} \%$ were fitted to a single exponential.

To assign the two values of the oxygen transport parameter to an appropriate phase, we assumed that the oxygen transport parameter in the $l_{0}$ phase was greater than that in the $s_{\mathrm{o}}$ phase and smaller than in the $l_{\mathrm{d}}$ phase. With this assumption, we accepted the commonly held statement that properties of the $l_{0}$ phase lay between those of gel- and fluid-phase membranes (Loura et al., 2001). We also applied another criterion to assess our results with coexisting phases. The properties of the $l_{\text {o }}$ phase that coexists below and above the phase-transition temperature with other phases should change gradually with increased temperature and without any abrupt change at the phase transition. In contrast, the properties
Single exponential mode

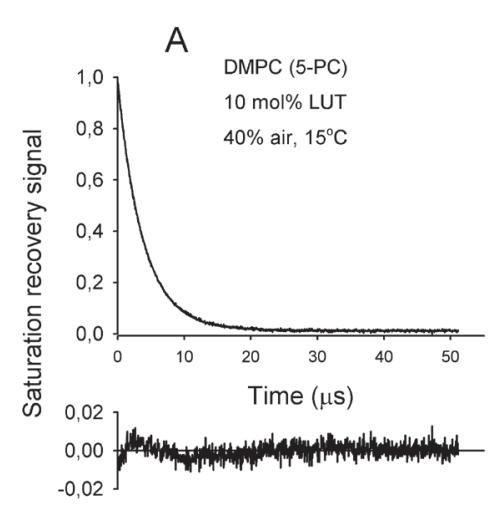

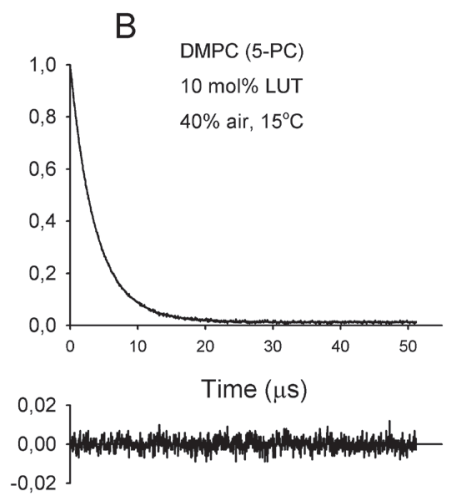

Double exponential mode
Figure 2. Representative SR signals with fitting curves and the residuals (the experim DMPC bilayer containing 10 mol\% lutein obtained at $15^{\circ} \mathrm{C}(\mathrm{A}$ and $\mathrm{B})$ and $35^{\circ} \mathrm{C}(\mathrm{C})$.

The liposome suspensions were equilibrated with the mixture of $40 \%$ air and $60 \%$ nitrogen. Experimental data were fitted either to single exponentials (A and C) or a double exponential (B). For SR signal presented in (A), the search for a single exponential fit was unsatisfactory (see the residual) while a double-exponential fit was excellent (see the residual in B). The double-exponential fit is consistent with the presence of two immiscible phases with different oxygen transport parameters. 

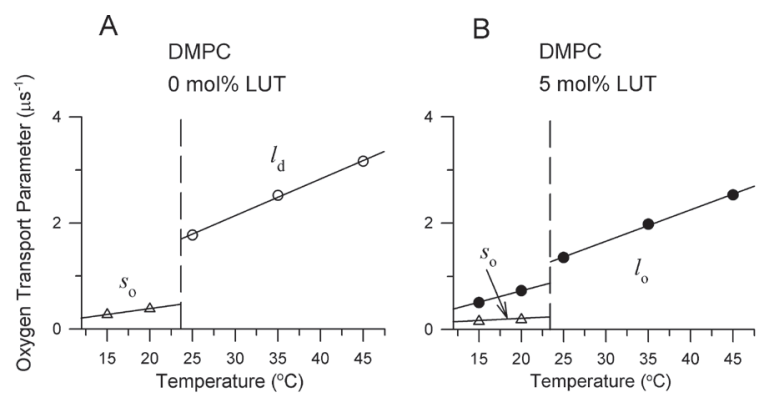

C DMPC
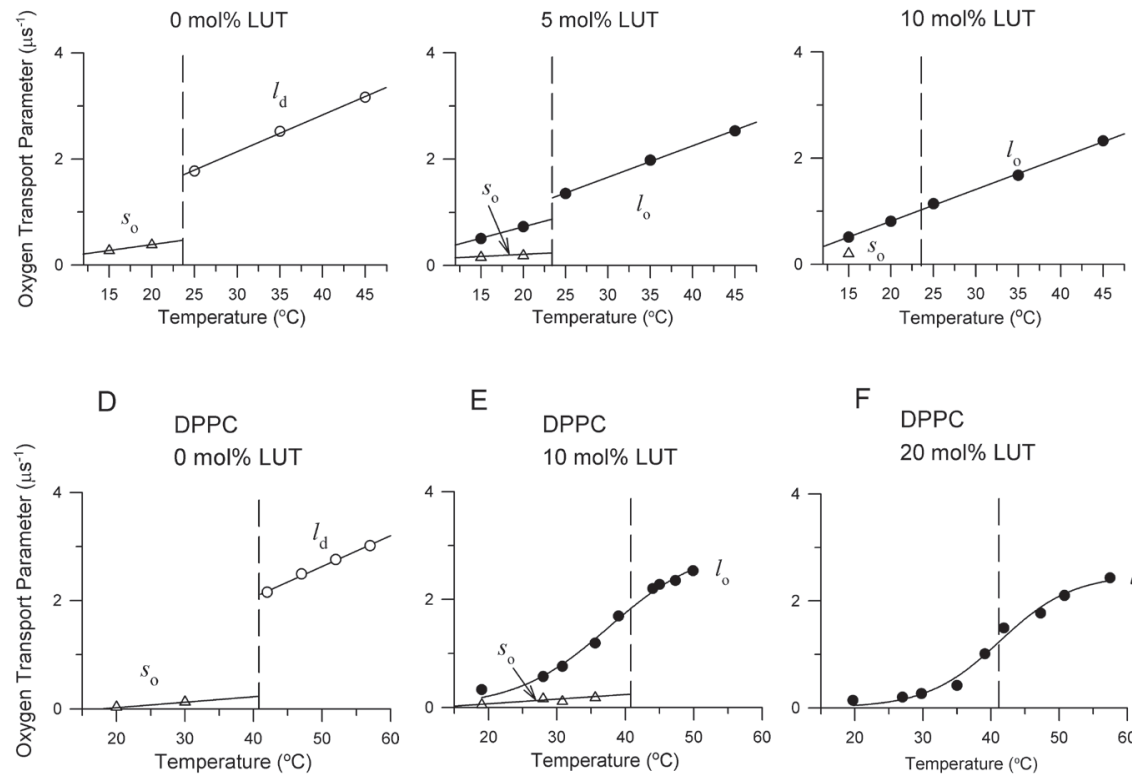

E DPPC

$10 \mathrm{~mol} \%$ LUT

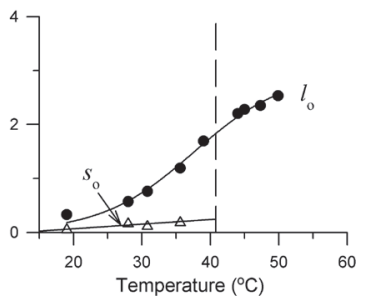

$\mathrm{F}$

DPPC

20 mol\% LUT

Figure 3. The oxygen transport parameter obtained with 5-PC in DMPC (A, B, C) and DPPC (D, E, F) membranes containing 0, 5, 10, and $20 \mathrm{~mol} \%$ lutein plotted as a function of temperature.

The vertical dashed lines at 23.6 and $41.2^{\circ} \mathrm{C}$ show the main phase-transition temperatures of DMPC and DPPC membranes, respectively. Data indicated that the lipid environment is homogenous in terms of oxygen transport for pure DMPC and DPPC $(s$ phase [ $\Delta$ ] below and $I_{\mathrm{d}}$ phase $[O]$ above the main phase-transition temperature) and in the presence of 5, 10, and 20 mol\% lutein above the phase-transition temperature $\left(I_{\mathrm{o}}\right.$ phase $\left.[0]\right)$. Both membranes containing 5 and 10 mol\% lutein exhibit two coexisting phases with different oxygen transport parameters below the phase-transition temperature. We attribute them to the $s_{0}(\Delta)$ and $I_{0}(\bullet)$ phase.
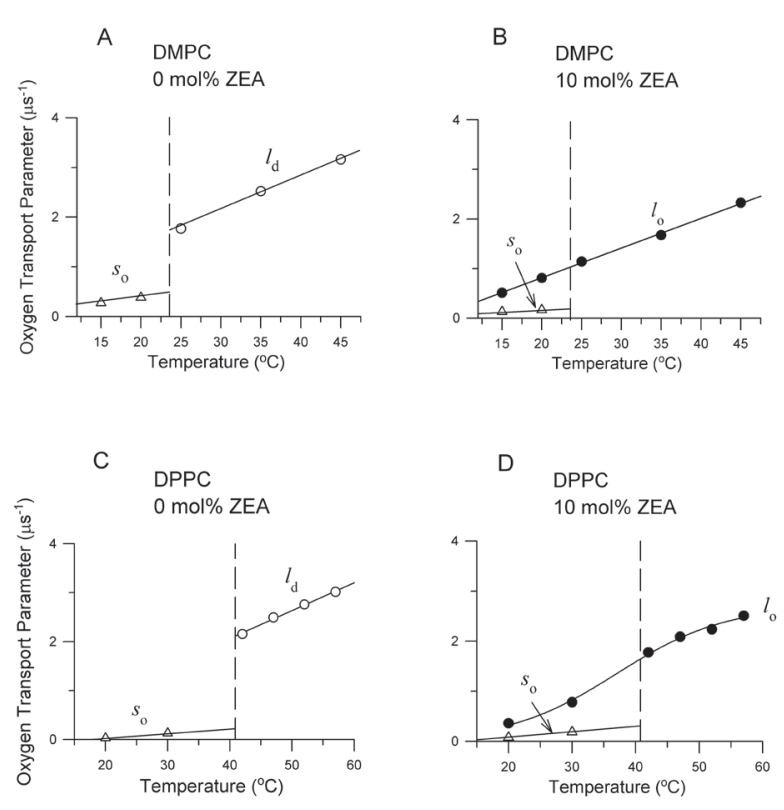

Figure 4. The oxygen transport parameter obtained with 5-PC in DMPC (A, B) and DPPC (C, D) membranes containing 0 and $10 \mathrm{~mol} \%$ zeaxanthin plotted as a function of temperature.

The vertical dashed lines at 23.6 and $41.2^{\circ} \mathrm{C}$ show the main phasetransition temperatures of DMPC and DPPC membranes, respectively. Data indicated that the lipid environment is homogenous in terms of oxygen transport for pure DMPC and DPPC ( $s$ phase $[\Delta]$ below and $l_{d}$ phase $[O]$ above the main phase-transition temperature) and in the presence of 10 mol\% zeaxanthin above the phase-transition temperature $\left(I_{\circ}\right.$ phase [0]). Both membranes containing $10 \mathrm{~mol} \%$ zeaxanthin exhibit two coexisting phases with different oxygen transport rates below the phase-transition temperature. We attribute these domains to the $s_{0}(\Delta)$ and $I_{0}(0)$ phase. of the $s_{\mathrm{o}}$ phase should change abruptly at the phase transition because this phase disappears at higher temperatures and becomes an $l_{\mathrm{d}}$ phase. Therefore, we performed careful measurements of temperature dependences of the oxygen transport parameter across the phase transition for DMPC and DPPC membranes containing different lutein and zeaxanthin concentrations.

\section{Oxygen transport parameter at the phase transition}

The oxygen transport parameter was measured as a function of temperature for DMPC and DPPC membranes containing 0, 5, 10, and $20 \mathrm{~mol} \%$ lutein or zeaxanthin in order to detect two purported phases existing below $\left(s_{\mathrm{o}}\right.$ and $\left.l_{\mathrm{o}}\right)$ and above $\left(l_{\mathrm{d}}\right.$ and $\left.l_{\mathrm{o}}\right)$ the phase-transition temperature of the membrane. These types of plots also help to decide which data points come from the $s_{\mathrm{o}}, l_{\mathrm{d}}$, and $l_{\mathrm{o}}$ phases (Subczynski et al., 2007b). Data shown in Figs. 3 and 4 indicate that the lipid environment is homogenous in terms of oxygen transport (only single-exponential SR signals were observed) for the pure DMPC and DPPC membranes $\left(s_{\mathrm{o}}\right.$ phase below and $l_{\mathrm{d}}$ phase above the phase-transition temperature), and in the presence of 5, 10, and 20 mol\% xanthophylls above the phase-transition temperature ( $l_{\mathrm{o}}$ phase). DMPC and DPPC membranes with overall xanthophyll mole fractions of 5 and 10 mol\% exhibit two coexisting phases, with different oxygen transport parameters only below the phasetransition temperature. We attribute them to $s_{\mathrm{o}}$ and $l_{\mathrm{o}}$. However, in the presence of $20 \mathrm{~mol} \%$ xanthophylls and below the phase-transition temperature (Fig. 3F), only one phase was found, which we attributed to $l_{0}$. Points assigned to the appropriate phases are indicated in Figs. 3 and 4. As shown above, points at which the oxygen transport parameter changes gradually across the phase transition were assigned to the 


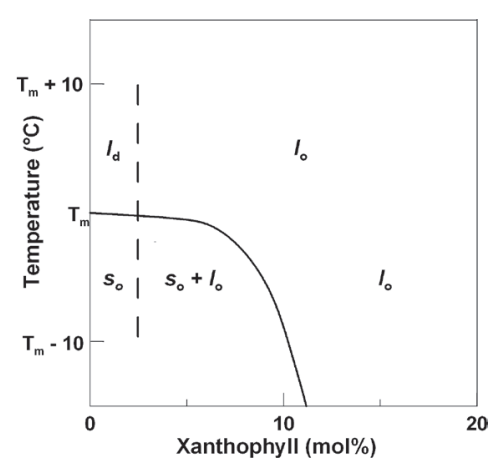

Figure 5. Generalized phase diagram for the PC-xanthophyll membranes. $T_{\mathrm{m}}$ indicates the phase-transition temperature for the PC membrane.

For details see discussion section.

$l_{\mathrm{o}}$ phase (below and above the phase-transition temperature); other points were assigned to the $s_{\mathrm{o}}$ phase below the phase-transition temperature and to the $l_{\mathrm{d}}$ phase above the phase-transition temperature. Such phase behavior was expected and is in agreement with the phase diagram proposed in the discussion section. Some of the discrepancies observed for the $l_{0}$ phase at $5 \mathrm{~mol} \%$ xanthophylls (Fig. 3B) may be a result of the redistribution of xanthophylls between coexisting $s_{\mathrm{o}}$ and $l_{\mathrm{o}}$ phases below the phase-transition temperature (determined by the related phase boundaries) and their concentration only in the sole $l_{0}$ phase at and above the phase-transition temperature.

The oxygen transport parameter in the $l_{0}$ phase, at all xanthophyll concentrations and at temperatures below the phase-transition temperature, is always greater than that in the pure gel-phase PC membrane and appropriate $s_{\mathrm{o}}$ phase. Above the phase-transition temperature, the oxygen transport parameter at all xanthophyll concentrations is always smaller in the $l_{\mathrm{o}}$ phase than in the $l_{\mathrm{d}}$ phase in pure DMPC and DPPC membranes. As expected, an abrupt change in the oxygen transport parameter (approximately, a four-times increase) was observed in the absence of xanthophylls at the main phase-transition temperature.

\section{Location of phase boundaries}

As a rule, in the presence of xanthophylls, the phase transition of PC membranes broadens and shifts to lower temperatures (Subczynski et al., 1993, Wisniewska et al., 2006, Widomska et al., 2009; Kolev \& Kafalieva, 1986). The shift is as great as about $3^{\circ} \mathrm{C}$, and the phase transition disappears at $\sim 10 \mathrm{~mol} \%$ xanthophyll concentration. Thus, these phase-transition temperatures form the horizontal phase boundary between the gel-phase membrane containing $s_{0}$ and $l_{0}$ phases and the fluid-phase membranes containing $l_{\mathrm{d}}$ and $l_{\mathrm{o}}$ phases. The vertical phase boundary lies close to $10 \mathrm{~mol} \%$ xanthophyll concentration. Below the phasetransition temperature and for xanthophyll concentrations smaller than $10 \mathrm{~mol} \%$ xanthophyll, two coexisting phases are observed (except with $10 \mathrm{~mol} \%$ lutein in DMPC membranes at $\left.20^{\circ} \mathrm{C}\right)$. The position of this vertical phase boundary is similar to the position of the corresponding boundary in PC-cholesterol membranes, assuming that the one molecule of xanthophyll exerts a similar effect on membrane organization as two molecules of cholesterol. Only these two phase boundaries can be determined based on the data presented here and in the literature. Also, based on our data, it can be said that the vertical phase boundary is nonexistent at about $10 \mathrm{~mol} \%$ xanthophyll concentration above the phase-transition temperature. Starting these experiments, we expected that this boundary would separate the coexisting $l_{\mathrm{d}}$ and $l_{\mathrm{o}}$ phases from the sole $l_{0}$ phase. Because we detected only the $l_{\mathrm{o}}$ phase above the phase-transition temperature, we believe the vertical phase boundary does not exist. Above the phase-transition temperature, xanthophyll molecules are uniformly distributed within the PC bilayer, forming a homogeneous phase.

\section{DISCUSSION}

Based on our measurements and data from the literature, we have proposed a phase diagram for PCxanthophyll membranes (Fig. 5) in which we indicate two regions: Region I, with the single $l_{0}$ phase (above and below the phase-transition temperature), and Region II, with coexisting $s_{\mathrm{o}}$ and $l_{\mathrm{o}}$ phases (below the phase-transition temperature). The horizontal boundary between Regions I and II was determined by measurement of the phase-transition temperature. The vertical boundary between Regions I and II was based on measurements of the oxygen transport parameter.

The most unexpected finding was the lack of two coexisting phases in the DMPC and DPPC membranes above the phase-transition temperature. This is a striking difference with PC membranes containing an appropriate amount of cholesterol for which two coexisting phases - namely, the $l_{\mathrm{d}}$ and $l_{\mathrm{o}}$ phases - were observed above the phase-transition temperature (Subczynski et al., 2007b; Wisniewska \& Subczynski, 2008). However, we see no conflict between the Gibbs' phase rule and the phase diagram presented in Fig. 5. The phase boundary between the $s_{\mathrm{o}}+l_{\mathrm{o}}$ region and the $l_{\mathrm{d}}$ $+l_{\mathrm{o}}$ region should have been at a constant temperature and not tilted (as was the case for the DMPC-cholesterol membrane phase diagram (Almeida et al., 1992). Our measurements and data from the literature indicate that this boundary is tilted (xanthophylls decrease the phase-transition temperature). Thus, according to the Gibbs' phase rule, it can separate regions containing no more than two phases: the $s_{\mathrm{o}}+l_{\mathrm{o}}$ region and the $l_{\mathrm{o}}$ phase.

Almeida et al. (1992) showed another phase boundary in Regions I and II for PC-cholesterol membranes at low cholesterol concentrations (about $5 \mathrm{~mol} \%$ ) (i.e., a boundary between the $l_{\mathrm{d}}$ phase and the region of the coexisting $l_{\mathrm{o}}$ and $l_{\mathrm{d}}$ phases, and a boundary between the $s_{\mathrm{o}}$ phase and the region of the coexisting $l_{\mathrm{o}}$ and $s_{\mathrm{o}}$ phases). Based on the above information and assuming that one molecule of xanthophyll exerts a similar effect on membrane organization as two molecules of cholesterol, we indicated similar boundaries in a very approximate manner for PC-xanthophyll membranes and positioned them at $2.5 \mathrm{~mol} \%$ xanthophyll concentration (Fig. 5, vertical dotted line). The xanthophyll solubility threshold should depend on many parameters including the method of membrane preparation, lipid type, the length and degree of unsaturation of alkyl chains, the presence of charges on the lipid headgroup, and inter-headgroup hydrogen bonds. However, it is not possible to control these conditions in different experiments and laboratories. To avoid these problems, we refrained from extending the phase diagram in Fig. 5 above $20 \mathrm{~mol} \%$ xanthophyll concentration. 


\section{Acknowledgements}

This work was supported by grants EY015526, EB002052, and EB001980 of the National Institutes of Health.

\section{REFERENCES}

Almeida PFF, Vaz WLC, Thompson TE (1992) Lateral diffusion in the liquid phases of dimyristoylphosphatidylcholine/cholesterol bilayers: a free volume analysis. Biochemistry 31: 6739-6747.

Almeida PFF, Pokorny A, Hinderliter A (2005) Thermodynamics of membrane domains. Biochim Biophys Acta 1720: 1-13.

Ashikawa I, Yin J-J, Subczynski WK, Kouyama T, Hyde JS, Kusumi A (1994) Molecular organization and dynamics in bacteriorhodopsinrich reconstituted membranes: discrimination of lipid environments by the oxygen transport parameter using a pulse ESR spin-labeling technique. Biochemistry 33: 4947-4952.

Bone RA, Landrum JT, Mayne ST, Gomez CM, Tibor SE, Twaroska EE (2001) Macular pigment in donor eves with and without AMD: a case-control study. Invest Ophthalmol Vis Sci 42: 235-240.

Britton G (1995) Structure and properties of carotenoids in relation to function. FASEB J 9: 1551-1558.

Chamberlain NR, Mehrtens BG, Xiong Z, Kapral FA, Boardman JL, Rearick JI (1991) Correlation of carotenoid production decreased membrane fluidity and resistance to oleic acid killing in Staphylococcus aureus 18Z. Infect Immun 59: 4332-4337.

Chattopadhyay MK, Jagannadham MV, Vairamani M Shivaji S (1997) Carotenoid pigments of an Antarctic psychrotrophic bacterium Micrococcus roseus: temperature-dependent biosynthesis structure and interaction with synthetic membranes. Biochem Biophys Res Commun 239: 85-90.

Gruszecki WI, Strzalka K (2005) Carotenoids as modulators of lipid memrane physical properties. Biochim Biophys Act 1740: 108-115.

Hyde JS, Subczynski WK (1989) Spin-label oximetry in: Biological magnetic resonance. Spin labeling: theory and applications. Berliner LJ, Reuben J, eds, vol 8, pp 399-425. Plenum New York.

Jagannadham MV, Chattopadhyay MK, Subbalakshmi C, Vairamani M, Narayanan K, Rao CM, Shivaji S (2000) Carotenoids of an Antartic psychrotolerant bacterium Sphingobacterium antarticus and a mesophilic bacterium Sphingobacterium multivorum. Arch Microbiol 173: 418-424.

Kawasaki K, Yin J-J, Subczynski WK, Hyde JS, Kusumi A (2001) Pulse EPR detection of lipid exchange between protein-rich raft and bulk domains in the membrane: Methodology development and its application to studies of influenza viral membrane. Biophys J 80: 738-748.

Kolev VD, Kafalieva DN (1986) Miscibility of beta-carotene and zeaxanthin with dipalmitoylphosphatidylcholine in multilamellar vesicles: a calorimetric and spectroscopic study. Photobiochem Photobiophys 11: 257-267.

Kusumi A, Subczynski WK, Hyde JS (1982) Oxygen transport parameter in membranes as deduced by saturation recovery measurements of spin-lattice relaxation times of spin labels. Proc Natl Acad Sci USA 79: 1854-1858.

Kusumi A, Subczynski WK, Pasenkiewicz-Gierula M, Hyde JS, Merkle H (1986) Spin-label studies on phosphatidylcholine-cholesterol membranes: effects of alkyl chain length and unsaturation in the fluid phase. Biochim Biophys Acta 854: 307-317.

Krinsky NI (2002) Possible biologic mechanisms for a protective role of xanthophylls. J Nutr 132: 540S-542S.

Krinsky NI Landrum JT, Bone R (2003) Biologic mechanisms of the protective role of lutein and zeaxanthin in the eye. Annu Rev Nut 23: $171-201$.

Landrum JT, Bone RA, Joa H, Kilburn MD, Moore LL, KE Sprague (1997) A one year study of the macular pigment: the effect of 140 days of a lutein supplement. Exp Eye Res 63: 57-62.

Loura LMS, Fedorov A, Prieto M (2001) Fluid-fluid membrane microheterogenity: a fluorescence resonance energy transfer study. Biophys J 80: 778-788.

Mainali L, Feix JB, Hyde JS, Subczynski WK (2011) Membrane fluidity as deduced by saturation-recovery EPR measurements of spinlattice relaxation time of spin labels. J Magn Reson 212: 418-425.

McIntosh TJ (1978) The effect of cholesterol on the structure of phosphatidylcholine bilayers. Biochim Biophys Acta 513: 43-58.

Pasenkiewicz-Gierula M, Subczynski WK, Kusumi A (1990) Rotational diffusion of a sterol molecule in phosphatidylcholine-cholesterol membranes: fluid-phase microimmiscibility in unsaturated phosphatidylcholine-cholesterol membranes. Biochemistry 29: 4059-4069.

Pintea A, Rugina DO, Pop R, Bunea A, Socaciu C (2011) Xanthophylls protect against induced oxidation in cultured human retinal pigment epithelial cells. J Food Comp Anal 24: 830-836.
Recktenwald DJ, McConnell HM (1981) Phase equilibria in binary mixture of phosphatidylcholine and cholesterol. Biochemistry 20: 45054510.

Rohmer M, Bouvier P, Ourisson G (1979) Molecular evolution of biomembranes: structural equivalents and phylogenetic precursors of sterols. Proc Natl Acad Sci USA 76 847-851.

Rottem S Markowitz O, (1979) Carotenoids acts as reinforcers of the Acholeplasma laidlawii lipid bilaver. I Bacteriol 140: 944-948.

Shimshick EJ, McConnell HM (1973) Lateral phase separation in phospholipid membranes. Biochemistry 12: 2351-2360.

Subczynski WK, Kusumi A (1986) Effects of very small amounts of cholesterol on gel-phase phosphatidylcholine membranes. Biochim Biophys Acta 854: 318-320.

Subczynski WK, Hyde JS, Kusumi A (1989) Oxygen permeability of phosphatidylcholine-cholesterol membranes. Proc Natl Acad Sci USA 86: 4474-4478.

Subczynski WK, Hyde JS, Kusumi A (1991a) Effect of alkyl chain unsaturation and cholesterol intercalation on oxygen transport in membranes: a pulse ESR spin labeling study. Biochemistry 30: 85788590 .

Subczynski WK, Markowska E, Sielewiesiuk J (1991b) Effect of polar carotenoids on the oxygen diffusion-concentration product in lipid bilayers An EPR spin label study. Biochim Biophys Acta 1068: 68-72.

Subczynski WK, Markowska E, Gruszecki WI, Sielewiesiuk J (1992) Effects of polar carotenoids on dimyristoylphosphatidylcholine membranes: spin-label studies. Biochim Biophys Acta 1105: 97-108.

Subczynski WK, Markowska E, Sielewiesiuk J (1993) Spin-label studies on phosphatidylcholine-polar carotenoid membranes: effects of alkyl chain length and unsaturation. Biochim Biophys Acta 1150: 173-181.

Subczynski WK, Felix CC, Klug CS, Hyde JS (2005) Concentration by centrifugation for gas exchange EPR oximetry measurements with loop-gap resonators. J Magn Reson 176: 244-248.

Subczynski WK, Widomska J, Wisniewska A, Kusumi A (2007a) Saturation-recovery electron paramagnetic resonance discrimination by oxygen transport (DOT) method for characterizing membrane domains in: Methods in Molecular Biology: Lipid Rafts. McIntosh TJ, ed, pp 145-159. Totowa: Humana Press.

Subczynski WK, Wisniewska A, Hyde JS, Kusumi A (2007b) Threedimensional dynamic structure of the liquid-ordered domain in lipid membranes as examined by pulse-EPR oxygen probing. Biophys $J$ 92: 1573-1584.

Subczynski WK, Widomska J (2009) EPR spin labeling in carotenoidsmembrane interactions in: Carotenoids Physical Chemical And Biological Functions and Properties. Landrum TJ, ed, pp 189-212. Boca Raton FL: CRC Press Taylor \& Francis Group.

Vist MR, Davis JH (1990) Phase equilibria of cholesterol dipalmitoylphosphatidylcholine mixtures. 2H-nuclear magnetic resonanceand differential scanning calorimetry. Biochemistry 29: 451-464.

Widomska J, Kostecka-Gugała A, Latowski D, Gruszecki WI, Strzałka K (2009) Calorimetric studies of the effect of cis-carotenoids on the thermotropic phase behavior of phosphatidylcholine bilayers. Biophys Chem 140: 108-114.

Wisniewska A, Subczynski WK (1998) Effects of polar carotenoids on the shape of the hydrophobic barrier of phospholipids bilayer. Biochim Biophys Acta 1368: 235-246.

Wisniewska A, Subczynski WK (2006a) Accumulation of macular xanthophylls in unsaturated membrane domains. Free Radic Biol Med 40: 1820-1826.

Wisniewska A, Subczynski WK (2006b) Distribution of macular xanthophylls between domains in model of photoreceptor outer segment membranes. Free Radic Biol Med 4: 1257-1265.

Wisniewska A, Widomska J, Subczynski WK (2006c) Carotenoid-membrane interactions in liposomes: effect of dipolar monopolar and nonpolar carotenoids. Acta Biochim Pol 53: 475-484.

Wisniewska A, Subczynski WK (2008) The liquid-ordered phase in sphingomyelincholesterol membranes as detected by the discrimination by oxygen transport (DOT) method. Cell Mol Biol Lett 13: 430-451.

Wrona M, Rozanowska M, Sarna T (2004) Zeaxanthin in combination with ascorbic acid or alpha-tocopherol protects ARPE-19 cells against photosensitized peroxidation of lipids. Free Radic Biol Med 36: 1094-1101.

Yin J-J, Feix JB, Hyde JS (1990) Mapping of collision frequencies for stearic acid spin labels by saturation-recovery electron paramagnetic resonance. Biophys J 58: 713-720.

Yin J-J, Pasenkiewicz-Gierula M, Hyde JS (1987) Lateral diffusion of lipids in membranes by pulse saturation recovery electron spin resonance. Proc Natl Acad Sci USA 84: 964-968.

Yin J-J, Subczynski WK (1996) Effects of lutein and cholesterol on alkyl chain bending in lipid bilayers: a pulse electron spin resonance spin labeling study. Biophys J 71: 832-839. 